Article

\title{
Cavitation simulation and mechanism analysis of underwater jet of cleaning equipment for aquaculture Fishing net
}

\author{
Taiping Yuan ${ }^{1 *}$, Xiaohua Huang ${ }^{1,2,3}, \mathrm{YU} \mathrm{Hu}^{1,2,3}$, Shaoming Wang ${ }^{1,2,3}$, Haiyang Liu ${ }^{1}$, Qiyou Tao ${ }^{1,2,3}$ \\ and Gengxi Guo ${ }^{1,2,3}$ \\ 1 South China Sea Fisheries Research Institute, Chinese Academy of Fishery Sciences; scshuyu@163.com \\ (Y.H.); wangshaomin2013@163.com (S.M.W); liuhaiyang@scsfri.ac.cn (H.Y.L); huangx-hua@163.com (X.H.H); \\ scsggx@163.com (G.X.G) \\ 2 Key Laboratory of Open-Sea Fishery Development, Ministry of Agriculture, China \\ 3 Guangdong Cage Engineering Research Center, China \\ * Correspondence: ytp0720@qq.com (T.P.Y.)
}

\begin{abstract}
In order to reveal the mechanism and influencing factors of high pressure jet cavitation of nozzle in submerged environment, this study focused on the evolutionary process of cavitation bubbles and combined finite volume method and mixed multi-phase flow model to analyze the cavitation, velocity distribution and experimental cavitation intensity of fishing net cleaning equipment. Results show that the cavitation inception, growth and collapse mainly occurred in the peripheral region of the flow field. Ring-shaped cavitation erosion zone appeared on the test sample target. A lot of small dense erosion pits were densely distributed in the ring-shaped erosion zone, erosion marks were observed in the center. The cavitation erosion intensity was greatly affected by the nozzle structure. As the diameter of nozzle increased from $0.6 \mathrm{~mm}$ to $1 \mathrm{~mm}$, the maximum gas volume fraction increased by $8.53 \%$. The nozzle outlet enlargement angle greatly increased the cavitation intensity. The nozzle with an outlet angle of $30^{\circ}$ exhibited the optimal cavitation erosion performance. The cavitation volume fraction of the nozzle with short necking structure was slightly larger than that of the nozzle with long necking structure at the same level in the necking length rang of $3 \mathrm{~mm}$ to $7 \mathrm{~mm}$. In terms of the influence of nozzle structure on the cavitation erosion effect, the nozzle diameter $\mathrm{D}$ ranked the first, followed by the outlet angle $\alpha$, and the necking length $\mathrm{L}$ was at the last.
\end{abstract}

Keywords: Cavitation jet; Finite volume method; Structural parameters; Numerical Simulation

\section{Introduction}

Owing to great aquaculture capacity and good fish quality, open high-density polyethylene (HDPE) deep water net cages now represent the advanced mariculture level of the current world. Net cages are soaked in the water for a long time, which can induce the production and attachment of some biogroups such as a lot of algae and shellfish[1,2]. The increasing attachments hinder smooth interchange between water inside and outside the cages, thereby easily leading to the deterioration of water quality and oxygen deficit for fish in the cage. Meanwhile, because of the accumulation of contaminations, a series of pathogenic bacteria such as aeromonas hydrophila and seawater vibrio grow and reproduce in water, which can then trigger the outbreak of fish diseases and even seriously mass death of fish shoal[3,4]. Mechanization and automation of net cleaning will certainly become the final solution to the industrial problem. 
Micro-nozzle is a key component in net cleaning equipment. When high-pressure water flows through the abrupt-change cross-section along the pipeline, a low-pressure region is formed near the wall and fluid is fractured to form a cavitation nucleus. Next, the cavitation nuclei grow, accumulate and collapse in the fluid. High speed, high pressure and high temperature are produced in collapse, which can generate noise and materials damage so as to achieve the cleaning of the attachments on the net[5-7]. Currently, scholars from all over the world mainly laid the research emphasis on the characteristics of the flow field in the nozzle. Liang et al. employed fluid dynamics software to analyze the effects of the nozzle type (cosine nozzle, exponential-tube nozzle, cylindrical nozzle and conical nozzle) as well as the length of the conical nozzle $L$ and the wall surface roughness Ks on jet flow velocity and the distribution of cavitation volume fraction[8]. According to their results, cosine nozzles exhibited higher jet impact force and the wall roughness affected these four types of nozzles to varying degrees. Zhou et al. established ASTM jet cavitation test platform and investigated the jet form in the cavitation region of the erosion region by comparing experimental results with numerical simulation results[9]. Ring-shaped cavitation erosion region was observed on the sample surface, which also expanded towards the sample center and finally induced serious shedding of materials. Using image acquisition and processing system, Ezddin et al. examined the effects of various factors such as jet flow pressure, the nozzle's structural parameters and shape as well as the growth cycle of the cavitation cloud on dynamics of the cloud[10]. They found that the geometrical structure of the cloud developed in oscillatory pattern after accumulation, growth and shrinkage, which was related to the fluctuation of the inlet pressure as well as the interaction between jet flow and the surrounding environment. Moreover, the characteristic oscillation frequency of the cavitating jet was determined.

Based on the bubble motion model, Kewen et al. simulated the motion of bubbles in the jet flow when passing through the nozzle, and analyzed the effects of the confining pressure and the upstream flow rate on bubble transportation efficiency[11]. According to their research results on the bubble's dynamic motion process, they concluded that upstream pressure was a main influence factor of cavitation intensity. The increase of flow velocity directly promoted the enhancement of cavitation intensity; meanwhile, fluid viscosity and surface tension imposed slight effects on the nozzle's cavitation intensity. Peng et al. calculated two-phase mixed mean flow based on unsteady Reynolds mean flow Navier equation, and estimated gas-phase volume fraction of the cavitating bubble using the simplified Rayleigh-Plesset equation for the calculation of cavitation intensity [12]. YANG et al. simulated the fluid characteristics in the center nozzle under submerged environment based on full-cavitation model [13]. According to their results, when the diameter of the center body remained unchanged, there existed an optimal outlet angle at the nozzle exist that resulted in intensive cavitation phenomenon. The cavitating bubbles produced by the nozzle with greater diameter were easily settled while small cavitating bubbles mainly focused at the jet interface. $\mathrm{H}$ et al. tracked both discrete phase and continuous phase in the nozzle fluid using Mont particle tracking method and Reynolds mean equation, and concluded that the structure of the nozzle neck significantly affected the distribution of discrete phase, fluid line and the length of the reflow region[14]. F. Piscaglia. et al employed a multi-Phase Dynamic-VOF solver to model primary jet atomization and cavitation inside high-pressure fuel injectors in OpenFOAM, the solver extension includes curvature includes curvature effect in the interface tracking, calculation results demonstrate the conservation and preservation property and accuracy of the solver[15]. Dai X.Y. et al employed high-speed imaging technique to realize the internal flow visualization of nozzle, combined with the 
simulation of nozzle internal flow was carried out with fluent 18.0, it was found that cavitation was mainly induced by the throttling effect at the crevice between the needle and needle seat and the redirection of flow at the nozzle inlet[16].

In order to reveal the cavitation formation mechanism in the nozzle of the net cleaning equipment under submerged environment and the dominant factor of the structure on cavitation degree, this study started from cavitation evolution mechanism and selected k-kl-w turbulence/transition model and cavitation model for simulating the flow characteristics of the nozzle. By designing the nozzle cavitation test under submerged environment, the effects of the nozzle structure on the cavitation degree were investigated in depth.

\section{Bubble evolution and cavitation mechanism}

In actual engineering test and application, cavitation fracture always occurs at the fluid weak point. There exist two types of weak spots in the fluid. The first type is the transient micro gaps induced by thermal motion of molecules in the liquid, which then forms the cavitation nuclei necessary for the growth and fracture of macro cavitation bubbles. The other type of weak spots exist at the boundary between liquid and solid wall or the boundary between liquid and small suspended particles[17]. The existence of these weak spots provides prerequisite condition for the initiation of cavitation nuclei.

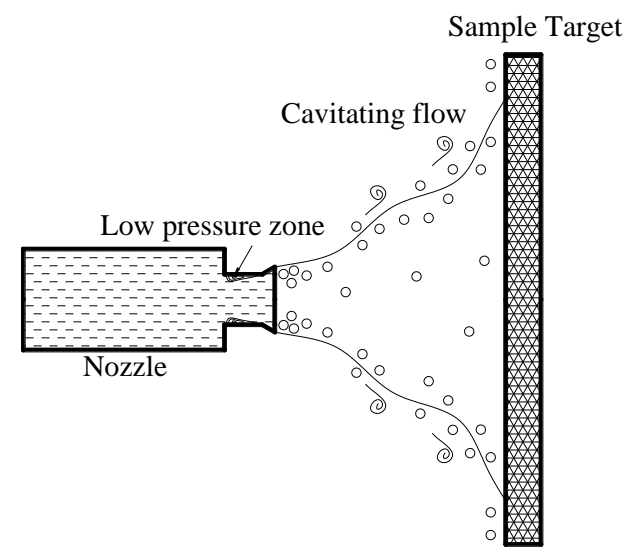

Figure 1. Schematic diagram of nozzle cavitation development process

The formation of low-pressure region is the necessary condition for the inception and growth of cavitation. As shown in Figure 1, high-pressure flow forms high-pressure jet at the nozzle's neck, and then, high-velocity jet is subjected to shear action by the static water on the nozzle's solid wall, thereby forming local low-pressure region at the interface between fluid and wall around the neck corner. When the pressure in the low-pressure region is lower than the saturated gas pressure $P_{v}$, the fluid is fractured, which triggers the formation of cavitation nuclei. The fracture generally occurs at the fluid weak spot. Meanwhile, high-pressure jet that enters into water environment can sweep the surrounding water and promote the formation of vorticity-concentrating region, and the pressure at the center of the vortex ring is far lower than the pressure in the other regions. Cavitation inception also appears in this type of vortexes. Any flowing fluid has certain cavitation number $\sigma$ [18]. $\sigma$ can be expressed in Equation (1):

$$
\sigma=\frac{P_{\infty}-P_{v}\left(T_{\infty}\right)}{\rho_{L} U_{\infty}^{2} / 2}
$$

where $P_{\infty}$ and $U_{\infty}$ denote $\mathrm{P}$ upstream pressure and velocity of the incoming flow, with unit of $\mathrm{Pa}$ and $\mathrm{m} / \mathrm{s} ; P_{\mathrm{v}}\left(T_{\infty}\right)$ denotes the vapor pressure of the liquid at the reference temperature $T_{\infty}$, with a 
unit of Pa. There exists a incipient cavitation number at a certain number $\sigma_{i}$. When $\sigma<\sigma_{i}$, cavitation nuclei are first formed at the liquid weak spots.

Assuming an infinitely homogeneous liquid region, the motion characteristics of a single bubble after cavitation inception are investigated. Based on the law of mass conservation, Raleigh derived the dynamic equation of cavitation bubbles without the consideration of fluid surface tension and viscosity item[19,20]. Rayleigh-Plesse equation can be written in Equation(2) :

$$
\frac{P_{\mathrm{B}}(t)-P_{\infty}(t)}{\rho_{L}}=R \frac{d^{2} R}{d t^{2}}+\frac{3}{2}\left(\frac{d R}{d t}\right)^{2}+\frac{4 v_{L}}{R} \frac{d R}{d t}+\frac{2 S}{\rho_{L} R}
$$

where $P_{B}(t)$ denotes the pressure in the cavitation bubble, $\rho_{L}$ denotes the fluid density, $R$ denotes the radius of the cavitation bubble and $S$ denotes the stress tensor.

When a cavitation bubble grows from a cavitation nucleus to several times of initial size, the bubble will be fractured at the maximum radius $R_{\mathrm{M}}$ (where $R_{\mathrm{M}}$ is approximately 100 times greater than the size of incipient cavitation nucleus). At the fracture moment, the nucleus is no longer spherical, and large-scale shock wave and micro-jet are produced at the fracture point in local fluid. When the fracture occurs on the surface of the underwater net, intensive disturbance can generate fairly high local transient surface stress. Under the repetitive pressure produced during the fracture of a great number of cavitation bubbles, the attachments on the net surface are crushed and fall off. Accordingly, the net can be cleaned based on cavitation mechanism [21-23]. The velocity, pressure and temperature at the moment of cavitation bubble fracture can be calculated in Equation(3-5):

$$
\begin{gathered}
\dot{R} \rightarrow-\left(\frac{R_{0}}{R}\right)^{\frac{3}{2}}\left[\frac{2\left(P_{\infty}^{*}-P_{v}\right)}{3 \rho_{L}}+\frac{2 S}{\rho_{L} R_{0}}-\frac{2 P_{G 0}}{3(k-1) \rho_{L}}\left(\frac{R_{0}}{R}\right)^{3(K-1)}\right]^{1 / 2} \\
P_{\text {max }}=P_{G 0}\left[(k-1)\left(P_{\infty}^{*}-P_{v}+3 S / R_{0}\right) / P_{G 0}\right]^{k /(k-1)} \\
T_{\max }=T_{0}\left[(k-1)\left(P_{\infty}^{*}-P_{v}+3 S / R_{0}\right) / P_{G 0}\right]
\end{gathered}
$$

where $\mathrm{k}$ is an approximation constant, $\mathrm{R}$ denotes the growth velocity of the cavitation bubble radius, $P_{\infty}^{*}$ denotes the constant value of the inflow pressure function $P_{\infty}(t)$ at $\mathrm{t}>0, P_{G 0}$ denotes the partial pressure of air in the bubble, $S$ denotes the fluid's surface tension and Ro denotes the initial radius of the cavitation nucleus. High-velocity flow, high temperature and high pressure during the fracture of the cavitation can induce noise and cause damages to materials. Accordingly, the underwater net cleaning equipment can use the impact force produced in constant fracture of bubbles so as to realize the cleaning of net at certain input pressure under different water pressures.

\section{Mesh generation of the computational domain}

\subsection{Geometrical structure of the nozzle}

In this study, the design parameters of the net cleaning equipment mainly include the pore diameter $d$, the necking length $l$, and the outlet angle $\alpha$. Figure 2 displays the nozzle structure. The nozzle center was made up of special ceramic core that was bonded with the $316 \mathrm{~L}$ stainless steel body by the industrial super core. The nozzle support was connected with the screw thread of the nozzle. In the present study, the net cleaning power was set as $18 \mathrm{~kW}$, the nominal flow was set as 50 $\mathrm{L} / \mathrm{min}$ and the maximum along-the-way pressure was set as $18 \mathrm{MPa}$. Based on the parameters 
settings of the cleaner, the pore diameter $d$ was set as $0.6 \sim 1.2 \mathrm{~mm}$, the inlet pressure was set as $6 \sim 15$ $\mathrm{MPa}$, the test flow was set as $22 \mathrm{~L} / \mathrm{min}$, thenecking length $l$ was set as $3 \sim 7 \mathrm{~mm}$ and the outlet angle $\alpha$ was set as $0 \sim 60^{\circ}$. The net-cleaning equipment had two discs and each disc included 4 nozzles.

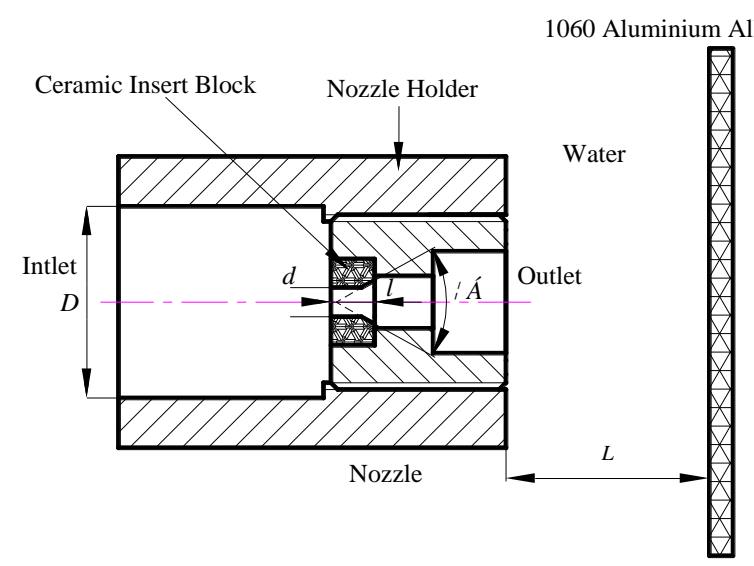

Notes: $D$ is the inlet diameter of the diameter, $\quad d$ is the diameter of the nozzle, $l$ is the length of the necking channel, $\alpha$ is the outlet angle of the nozzle, and $L$ is the target distance.

Figure 2. Geometric Model of Computational Domain

\subsection{Mesh generation and dependence validation}

For the convenience of numerical calculation, the geometrical model of the computational domain of the nozzle was first simplified. Using the pre-processing software (ICEM) in CFD, the computational domain of the nozzle was discretized as the structural network, as the generated meshes displayed in Figure 3. Moreover, the meshes around the neck were denser. The nozzle inlet was set as the computational inlet, the rotational central axis of the nozzle was set as the symmetrical axis of the computational domain, the nozzle wall and the boundary of the fluid domain were set as the wall of the computational domain, and the right boundary of the fluid domain was set as the outlet of the computational domain. The diameter of the nozzle inlet was $4 \mathrm{~mm}$, the diameter of the outlet of the computational domain was $40 \mathrm{~mm}$ and the total length of the computational domain was $50 \mathrm{~mm}$. The nozzle is rotatory in structure and half of the part around the symmetric axis was set as the computational domain.

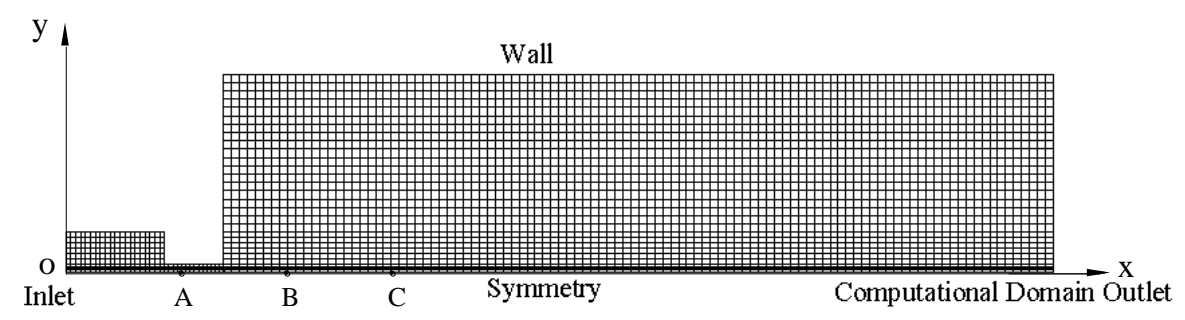

Figure 3. Grid generation of computational domain

In order to reduce the computational error, the number of the generated meshes should be determined. No relation between the time length and the calculated results should also be confirmed. As regard to the computational domain as shown in Figure 3, three points along the axial direction (specifically, A $(12,0), \mathrm{B}(20,0)$ and C $(30,0))$ were selected as the monitoring points, and four different types of mesh generation were designed, as listed in Table 1. Among these schemes, when the mesh number varied from $2.5 \times 10^{4}$ to $2.0 \times 10^{5}$, the computational model and the boundary conditions were same. Figure 4 displays the calculated results using different schemes. When the number of the meshes exceeded $1.17 \times 10^{5}$, the maximum deviation of the velocity of these three 
points along the axial direction was smaller than $1.8 \%$, in which the calculated velocity using Scheme 4 was used as the reference. By taking both computational period and precision into overall consideration, Scheme 2 was adopted in further analysis.

Table 1. Grid computation solution

\begin{tabular}{cccc}
\hline Schemes & Number of total elements & Number of planes & Number of Total nodes \\
\hline 1 & 25631 & 54206 & 28052 \\
2 & 117126 & 238199 & 121091 \\
3 & 160610 & 328470 & 166546 \\
4 & 204169 & 417278 & 211291 \\
\hline
\end{tabular}

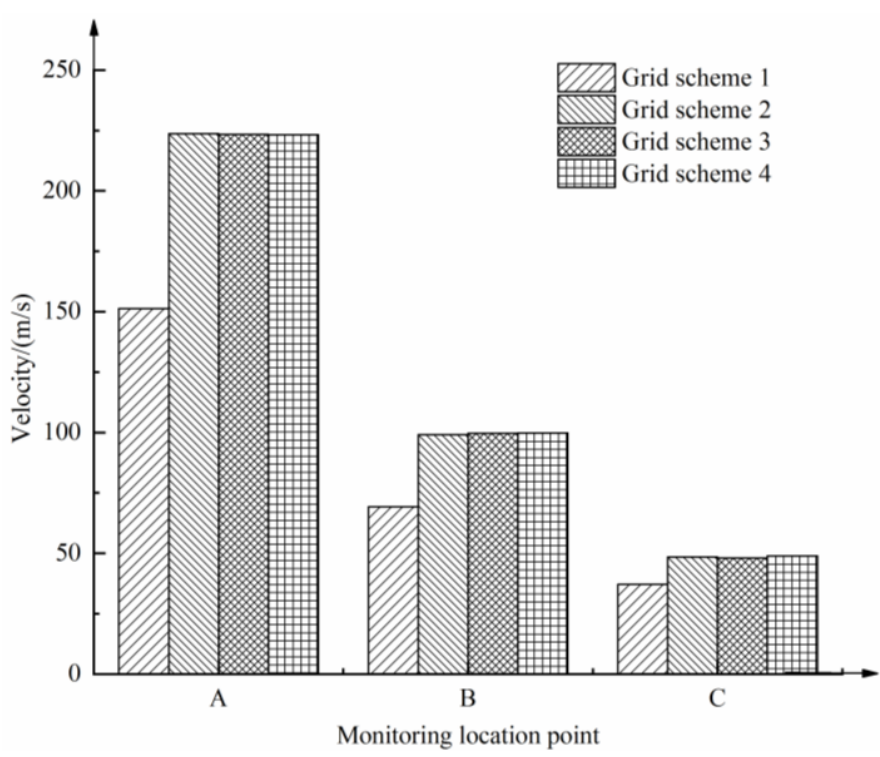

Figure 4. Verification results of grid independence

For the determined mesh generation scheme, in order to ensure sufficient iteration number in the computational domain and the reliability of the calculated results, the convergence standard of the monitoring parameter was set as $10^{-6}$, and the number of iterations was set as different value in each time step so that the parameter should be iterated for many times until reaching the convergence. The number of iterations was set as 500, 1,500, 2,500, 3,000 and 5,000, respectively. Fig. 5 displays the calculated result of the linear velocity of the symmetrical axis. When the number of the iterations exceeded 1,500, the distribution curves of the linear velocity of the central axis were almost coincident. It can thus be regarded that the calculation reached the stable state after the iterations over 1,500 times. Through overall consideration of computational period and precision, the number of iterations was set as 3,000 in this study. 


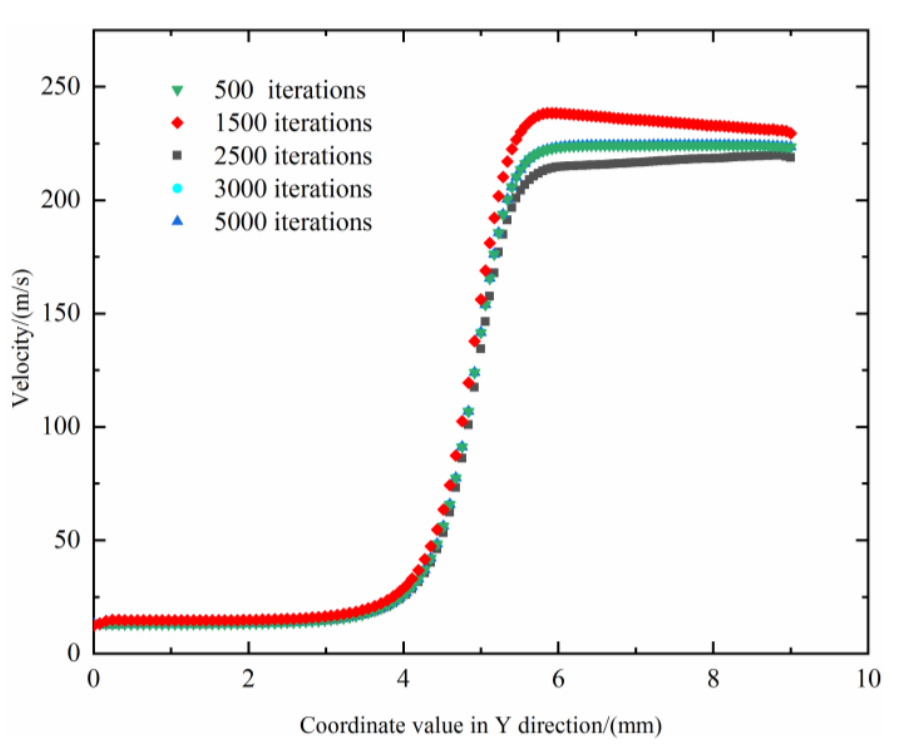

Figure 5. Influence of distribution of the axial velocity with number of iterartions

\section{Simulation and analysis of results}

The inlet and outlet boundary condition was set as pressure boundary. The inlet pressure and the outlet pressure were set as $12 \mathrm{MPa}$ and $0.1 \mathrm{MPa}\left(P_{\text {in }}=12 \mathrm{MPa}\right.$ and $\left.P_{\text {out }}=0.1 \mathrm{MPa}\right)$, respectively. Under room temperature, the saturated vapor pressure was $3540 \mathrm{~Pa}\left(P_{\mathrm{v}}=3540 \mathrm{~Pa}\right) . k-k l-w$ turbulence model and Zwart-Ger-Belamri cavitation model were selected. The solving mode was set as 'Coupled Solver'. The discretization pattern was set as 'QUICK' and the pressure difference pattern was set as 'PRESTO'[24, 25]. Figure 6 displays the simulated velocity distribution images.

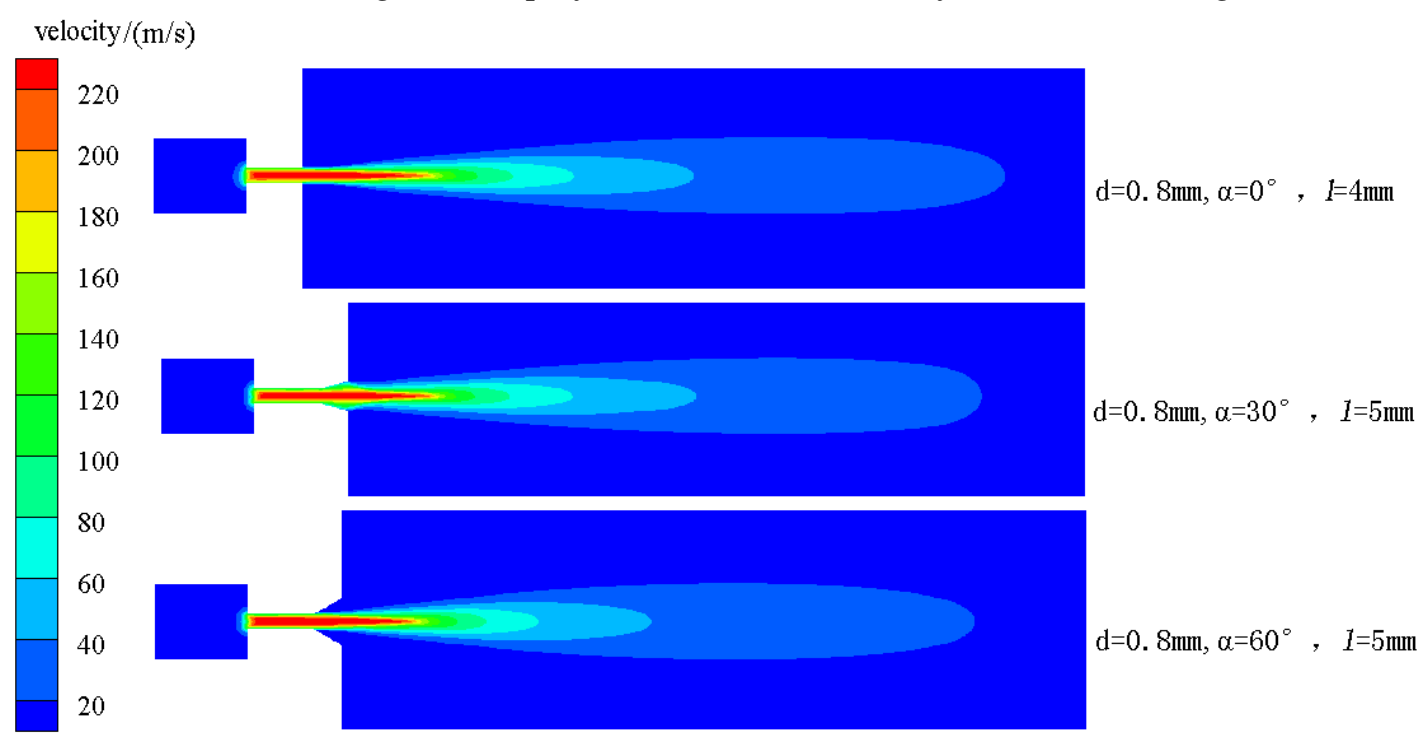

Notes: aspect ratio $n=d / l, l$ is the length of nozzle necking channel

Figure 6. Diameter $0.8 \mathrm{~mm}$ Nozzle velocity nephogram

\subsection{Effect of the nozzle diameter $d$ on the cavitation distribution}

Figure 7 displays the distribution of cavitation volume fraction on the sample surface at a distance $L$ from the nozzle outlet, in which $d=0.6 \sim 1.2 \mathrm{~mm}, \alpha=0^{\circ}$ and $l=5 \mathrm{~mm}$. When the target distance $L$ remained unchanged, the maximum cavitation volume fraction and the cavitation region area of the nozzle with a diameter of $1.2 \mathrm{~mm}(d=1.2 \mathrm{~mm})$ was greatest among these four nozzles. Meanwhile, the maximum cavitation volume fraction was located outside the high-pressure jet, and the 
distribution curve of the cavitation volume fraction exhibited symmetrical distribution around the maximum value axis. Overall, with the increase of the nozzle diameter, both the maximum cavitation volume fraction and the cavitation region increased, thereby enhancing the cavitation efficiency. When $L>20 \mathrm{~mm}$, the cavitation volume fraction reached the minimum.

Under intensive shear action between high-velocity jet and the nozzle wall/the surrounding low-velocity fluid, several vorticity-concentrating regions were formed, and the pressure at the vortex center was far below than the values in other regions, thereby inducing the inception of cavitation. It can thus be found that the whole cavitation inception region was located in the peripheral region of the flow field. The increase of nozzle diameter can promote the shear action between high-velocity jet and the surrounding low-velocity fluid. The inception, growth and collapse of cavitation in the nozzle mainly occur in the peripheral region of the flow field. Accordingly, it can be predicted that annular cavitation erosion region appeared around the high-pressure jet. Under same inlet pressure, increasing the nozzle diameter was beneficial to enhancing the cavitation erosion intensity.

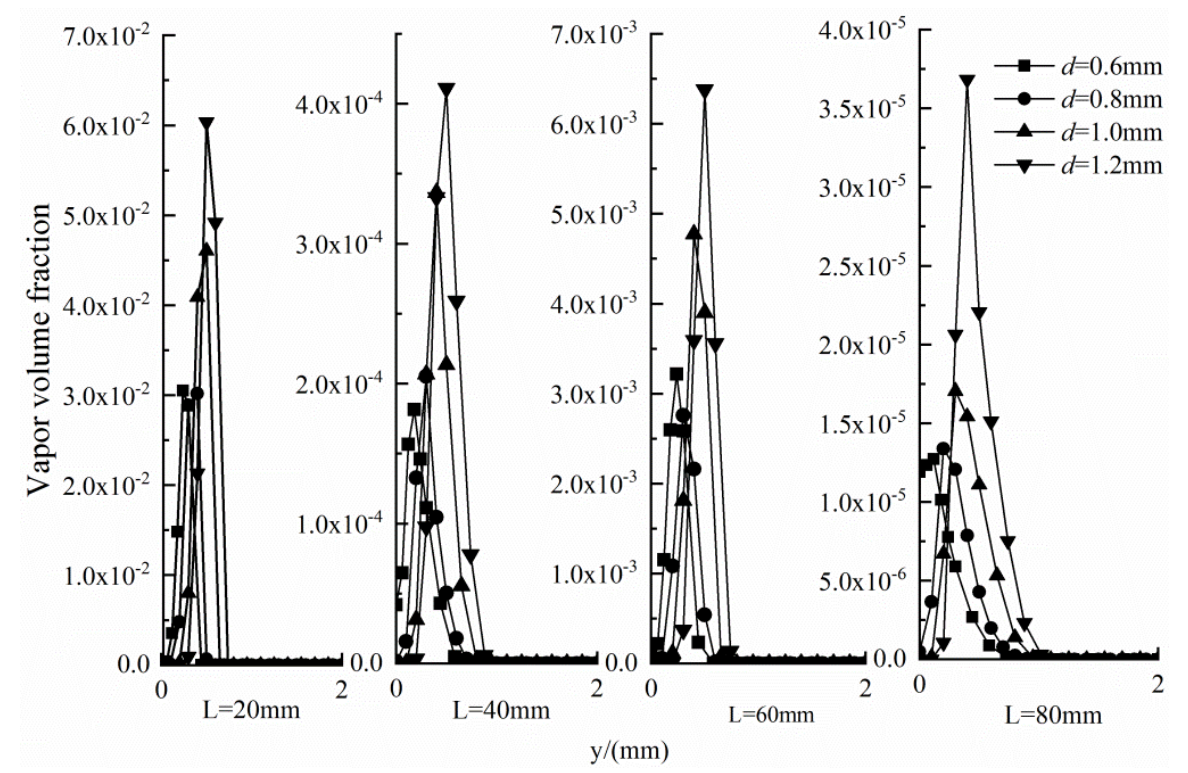

Figure 7. Vapor volume fraction of different diameters nozzles at target distance $L$

\subsection{Effect of the outlet expansion angle of the nozzle $\alpha$ on the jet distribution}

In this study, the computational region was the flowing liquid pipe model with constant pressure difference. The flow field in the nozzle can be approximated as a steady flow field. During the simulation, $d=0.8 \mathrm{~mm}, \alpha=0^{\circ}, l=5 \mathrm{~mm}$. Figure 8 displays the simulated results when the outlet angle of the nozzle was set as three different values. Overall, the flow field in the nozzle was almost unchanged, i.e., the change of the outlet angle imposed almost no effect on the distribution of flow field in the nozzle.

By comparing Figure 8(b) and Figure 8(c), backflow phenomena to varying degrees can be observed at the outlet corner of the nozzle. The formation of the backflow region was related to intensive shear action between high-velocity jet and the environmental fluid as well as wall attachment effect. The appearance of backflow region promoted the formation of multiple vorticity-concentrating regions. The pressure at the vortex center was far lower than the values in other regions. Under high-velocity flow action, the pressure at the vortex center dropped significantly, which led to the increase of gas bubbles in both number and degree. 


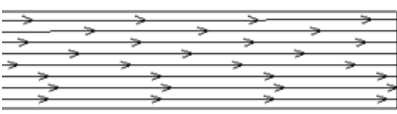

(a)

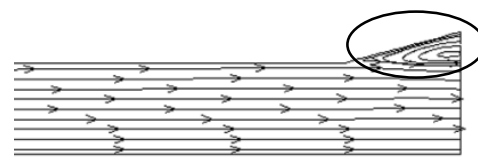

(b)

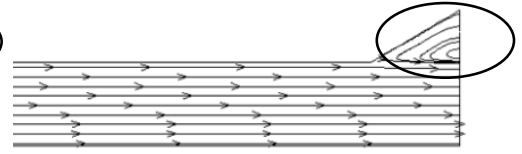

(c)

Figure 8. Velocity streamline diagram of nozzle with different angles outlet;(a) $\alpha=0^{\circ}$;(b) $\alpha=30^{\circ}$; (c) $\alpha=60^{\circ}$

Table. 2 displays the distributions of the flow velocity of the backflow region at the outlet along y-axis direction when using the nozzles with $\alpha=30^{\circ} \sim 60^{\circ}$, respectively. As described above, the backflow region was outside the neck radius. Using the nozzle with an outlet angle of $30^{\circ}$, flow velocity was $43.5 \%$ higher than the value using the nozzle with an outlet angle of $50^{\circ}$, suggesting a larger vorticity in the backflow region. Using these two nozzles, the pressure distribution at the vortex center followed identical rules. It can thus be predicted that, under same boundary condition, the cavitation degree of the nozzle with an outlet angle of $30^{\circ}$ was far greater than that of the nozzle with an outlet angle of $60^{\circ}$. In other words, the nozzle with an outlet nozzle of $30^{\circ}$ exhibited more favorable cleaning effect and cavitation erosion performance.

Table 2. Flow velocity Comparison at the reflow area of nozzle outlet

\begin{tabular}{cccccc}
\hline Coordinate value in Y direction & $\mathbf{0 . 4 4}$ & $\mathbf{0 . 4 6}$ & $\mathbf{0 . 4 8}$ & $\mathbf{0 . 5 0}$ & $\mathbf{0 . 5 2}$ \\
\hline Velocity of nozzle of outlet angle $30^{\circ}$ & 6.94456 & 7.5523 & 7.48836 & 7.32812 & 6.27064 \\
Velocity of nozzle of outlet angle $60^{\circ}$ & 3.90242 & 3.69445 & 3.3912 & 3.17912 & 3.17912 \\
ratio & $43.5 \%$ & $51.1 \%$ & $54.6 \%$ & $56.6 \%$ & $49.3 \%$ \\
\hline
\end{tabular}

\subsection{Effect of the necking length of the nozzle $\alpha$ on the jet distribution}

Figure 9 displays the distributions of the cavitation gas phase volume fractions at two cross-sections in the computational domain using three different types of nozzles. During the simulation, $d=0.8 \mathrm{~mm}, \alpha=0^{\circ}, l=3 \sim 7 \mathrm{~mm}$, while the necking length was set as different values (3 mm, 5 $\mathrm{mm}$ and $7 \mathrm{~mm}$ ). It can be observed that gas phase volume fraction in a same computational domain exhibited similar distribution patterns when using these three nozzles. At $1 \mathrm{~mm}$ away from the nozzle neck, the gas-phase volume fraction dropped steadily along the cross-section. At $1 \mathrm{~mm}$ away from the nozzle outlet, the gas volume fractions reached the maxima when these nozzles deviated from the rotating axis. This is consistent with the formation mechanism of cavitation. Using the nozzle with a necking length of $3 \mathrm{~mm}$, the gas-phase volume fraction reached a maximum of 0.375 at $1 \mathrm{~mm}$ away from the nozzle neck, which was 0.031 at $1 \mathrm{~m}$ away from the nozzle outlet. The gas-phase volume fractions when using the nozzle with a necking length of $3 \mathrm{~mm}$ exceeded the condition when using the other types of nozzles. Accordingly, it can be concluded that the increase of the nozzle's necking length can weaken the cavitation intensity at the corresponding position away from the nozzle neck. Similarly, as shown in Fig. 9(b), the nozzle with a smaller necking length exhibited greater cavitation volume fraction distribution in the external flow field when the inner hole diameter remained unchanged. At $1 \mathrm{~mm}$ from the nozzle neck, the range of cavitation volume fraction was 0.05624 ; at $1 \mathrm{~mm}$ away from the nozzle outlet, the range of the cavitation volume fraction was 0.00852. Overall, using these three nozzles, the cavitation volume fraction exhibited slight change, suggesting that the necking length at the nozzle neck imposed quite limited effect on the cavitation degree. 


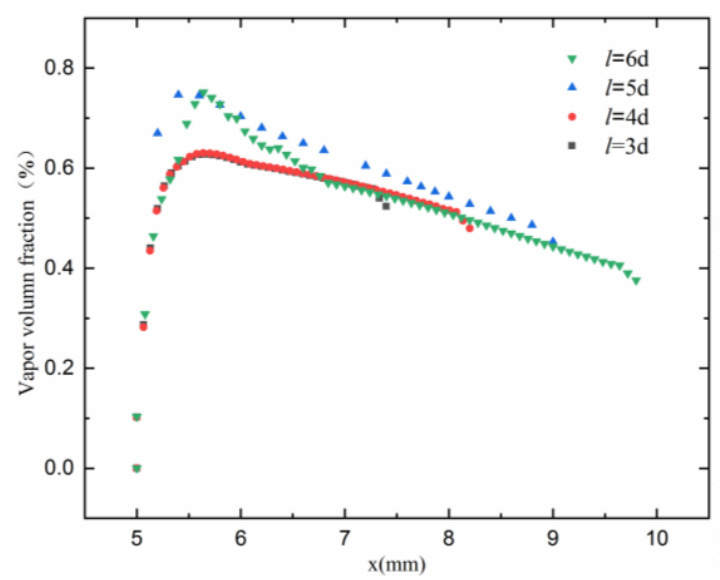

(a)

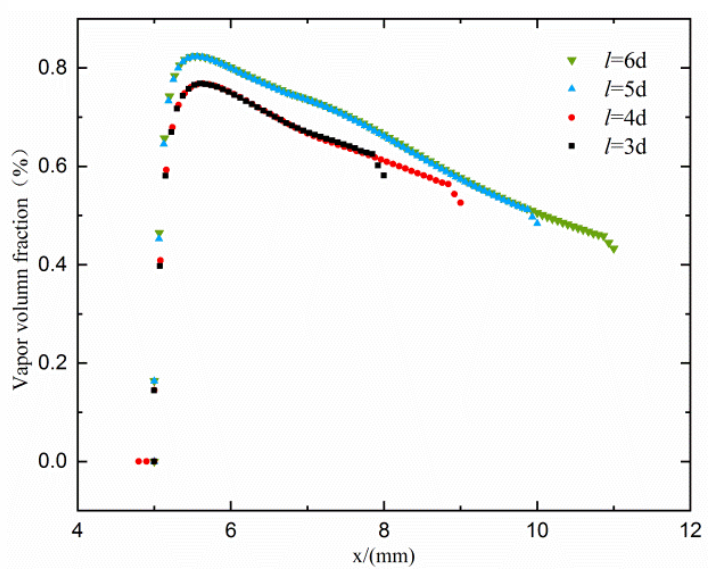

(b)

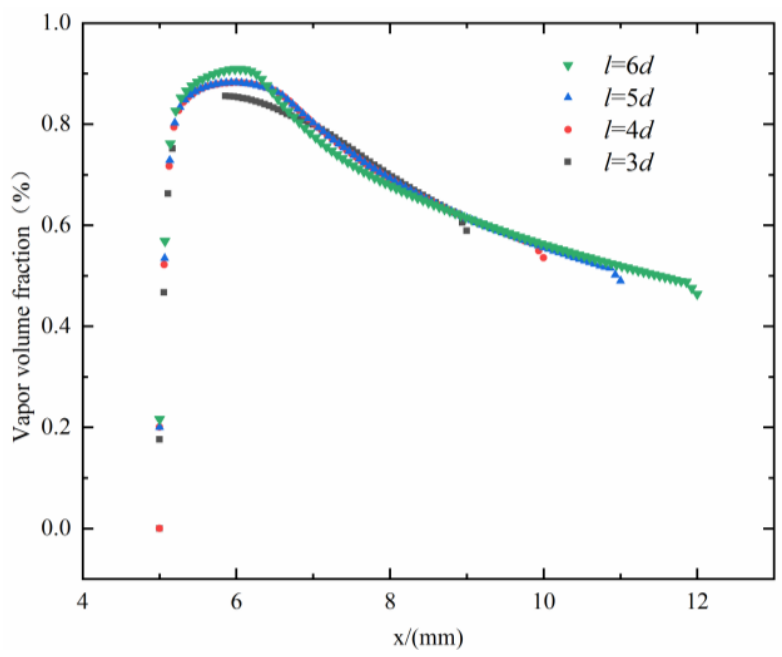

(c)

Figure 9. Effect of nozzle aspect ratio n on vapor volume fraction. (a) $d=0.8 \mathrm{~mm}$;(b), $d=1.0 \mathrm{~mm}$; (c) $d=1.2 \mathrm{~mm}$

\section{Test and analysis}

\subsection{Test equipment and method}

The detailed test method was detailedly described in Refs.[26-29], as the test system displayed in Fig. 10. It can be observed that the test equipment is mainly composed of the high-pressure pump of a gasoline engine, a regulating pump, a pressure gauge, valves, water tanks, nozzles and clamps, the flowmeter, and the sample target. The pressure of the high-pressure pump was set as $12 \mathrm{MPa}$. The sample target, with a size of $20 \mathrm{~mm} \times 20 \mathrm{~mm} \times 3 \mathrm{~mm}$, was manufactured by QGIII plasma cutter. The sample surface was first polished by metallographic abrasive papers, then cleaned by ethyl alcohol and dried During the test, the nozzle and clamps were located at $10 \mathrm{~cm}$ below water. 


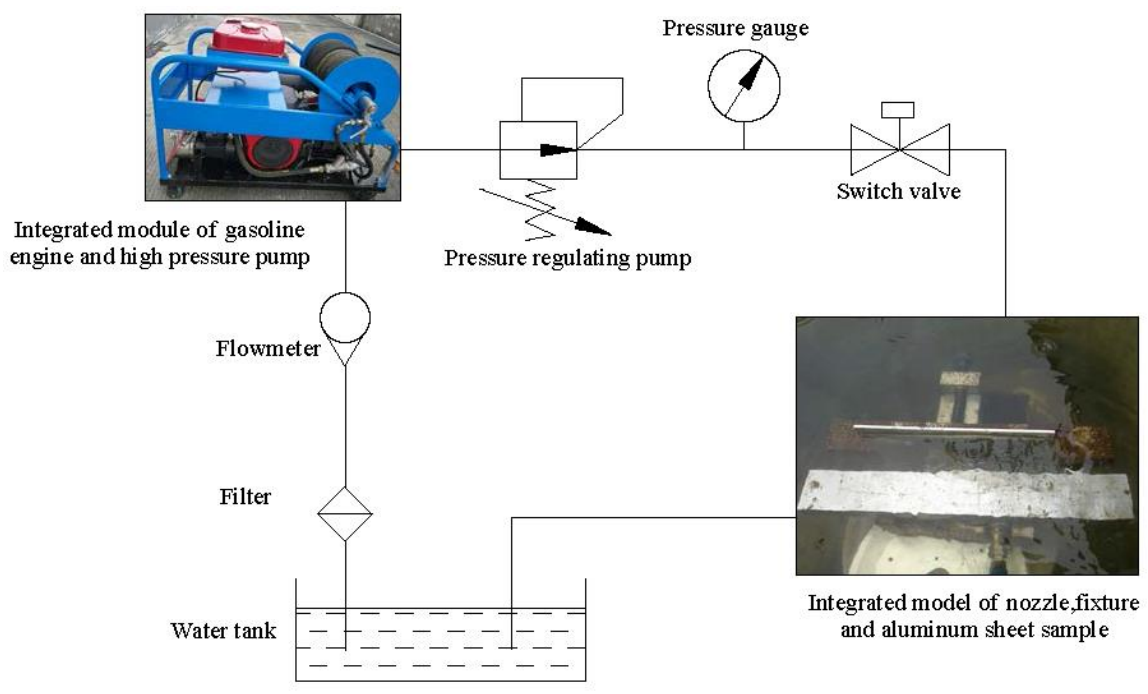

Figure 10. Diagram of experimental equipment

In order to gain more in-depth knowledge of the cavitation erosion characteristics of the nozzle, validate the simulation results and determine the engineering parameters of the nozzle, a $3 \times 3$ orthogonal test was designed, in which the pore diameter $D$, the outlet angle $\alpha$ and the necking length 1 were selected as the factors. Table 3 lists the levels of various factors. The reference values were set by taking into account both the technological processing feasibility and the commonly-used values. Fig. 4 displays the orthogonal test results.

Table 3. Three factors and three levels orthogonal table

\begin{tabular}{cccc}
\hline \multirow{2}{*}{ Levels } & \multicolumn{3}{c}{ Factors } \\
\cline { 2 - 4 } & Diameter D/ $(\mathbf{m m})$ & Outlet angle $\boldsymbol{\alpha} /\left(^{\circ}\right)$ & Necking length $\boldsymbol{l} /(\mathbf{m m})$ \\
\hline 1 & 0.6 & 0 & 3 \\
2 & 0.8 & 30 & 5 \\
3 & 1 & 60 & 7 \\
\hline
\end{tabular}

\subsection{Analysis of test results}

Figure 11 displays the cavitation erosion results. On account of limited space, only the test results of some representative samples are shown in Figure 11. Overall, the change of the nozzle parameters imposed significant effects on the sample's capitation effect. Under submerged environment, the cavitation erosion almost exhibited annular distribution patterns. The erosion pits were densely distributed in the annular erosion region and obvious erosion marks appeared at the dead centre. This is consistent with numerical simulation results, suggesting that water jet impact force was extremely reduced under submerged environment. The sample was mainly subjected to high-temperature and high-pressure action induced by the collapse of cavitation bubbles.

As shown in Figure 11 (a), (b) and (f), when the nozzle's pore diameter and necking length were fixed, the existence of the outlet angle can enhance the cavitation erosion under submerged environment, which also fit well with the numerical simulation results. In the meantime, the cavitation degree when using the nozzle with an outlet angle of $30^{\circ}$ far exceeded that when using the nozzle with an outlet angle of $60^{\circ}$ in terms of both erosion depth and range. This is in good consistency with the distribution rules of the vorticity in the backflow region. Accordingly, it can be concluded that the nozzle with an outlet angle of $30^{\circ}$ can more effectively promote the formation of 
cavitation nuclei and the growth of bubbles than the nozzle with an outlet of $60^{\circ}$, i.e., the outlet angle of $30^{\circ}$ is more applicable to underwater net cleaning equipment.

Figure 11(d) and Figure 11(e) represents the effect of the nozzle's pore diameter on cavitation effect. Under same inlet pressure, the diameter of the erosion ring using the nozzle with a pore diameter of $0.8 \mathrm{~mm}$ ranged from $4 \sim 14 \mathrm{~mm}$, while the diameter of the erosion ring using the nozzle with a pore diameter of $0.6 \mathrm{~mm}$ ranged from $7 \sim 12 \mathrm{~mm}$. However, the cavitation erosion intensity changed slightly with the pore diameter. Accordingly, it can be concluded that the change of nozzle diameter mainly affected the distribution range of the cavitation erosion range but slightly affected the cavitation erosion intensity.

Through comparison, the outer diameters of the cavitation erosion rings under two conditions $\left(D=0.8 \mathrm{~mm}, \alpha=30^{\circ}\right.$ and $\left.l=5 \mathrm{~mm}\right)$ and $(D=0.8 \mathrm{~mm}$ and $\mathrm{l}=3 \mathrm{~mm})$ were $11 \mathrm{~cm}$. The cavitation erosion intensity in Figure 11 e) was slightly greater than that in Fig. b). The radii of the cavitation erosion rings under two conditions $\left(D=0.8 \mathrm{~mm}, \alpha=30^{\circ}\right.$ and $\left.l=5 \mathrm{~mm}\right)$ and $\left(D=0.8 \mathrm{~mm}, \alpha=0^{\circ}\right.$ and $\left.l=7 \mathrm{~mm}\right)$ were $11 \mathrm{~cm}$. The cavitation erosion intensity of the sample target in Figure $11 \mathrm{f}$ ) was greater than that in Figure $11 \mathrm{c})$, suggesting that the cavitation degree of the nozzle with a necking length of $3 \mathrm{~mm}(l=3$ $\mathrm{mm}$ ) was slightly greater than that of the nozzle with $l=5 \mathrm{~mm}$, while the cavitation degree of the nozzle with $l=7 \mathrm{~mm}$ was weakest. In other words, the increase of the neck in the nozzle weakened the nozzle's cavitation effect to certain degree, which agrees well with the influence rules of the necking length on the cavitation intensity in the inner flow field.

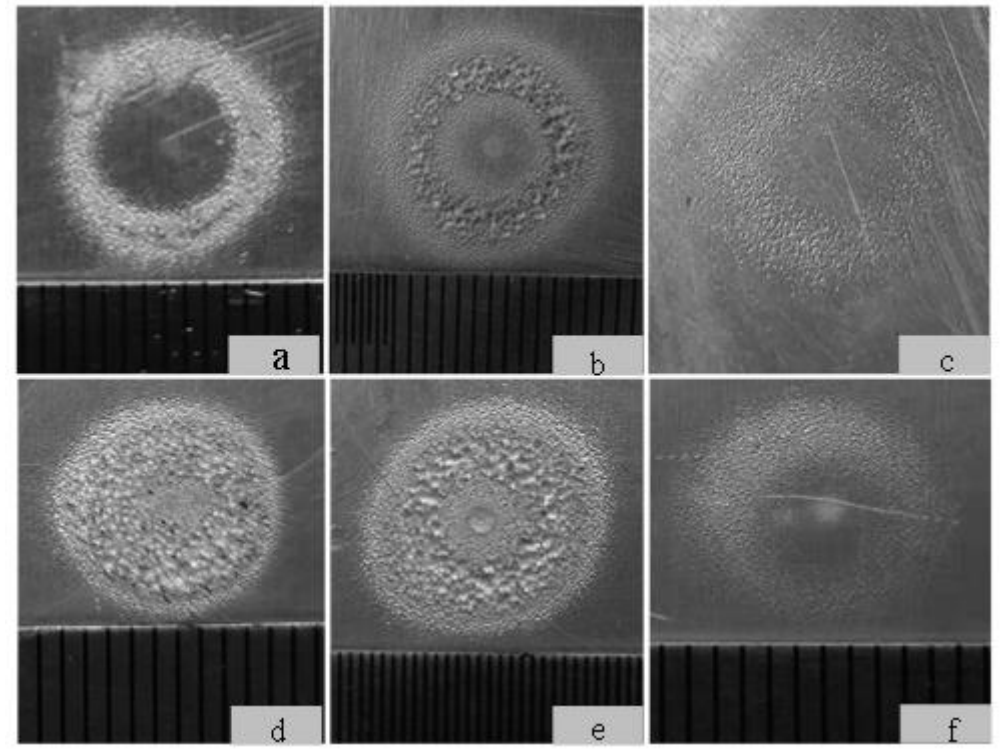

Figure 11. Cavitation results of samples with different nozzle structure parameters; a) $D=0.8 \mathrm{~mm}, \alpha=60^{\circ}, l=5 \mathrm{~mm}$; b) $D=0.8 \mathrm{~mm}, \alpha=30^{0}, \quad l=5 \mathrm{~mm} ; \quad$ c) $D=0.8 \mathrm{~mm}, \alpha=0^{0}, \quad l=7 \mathrm{~mm}$; (d) $D=0.6 \mathrm{~mm}, \alpha=30^{0}, l=3 \mathrm{~mm}$; (e) $D=0.8 \mathrm{~mm}, \alpha=30^{\circ}, l=3 \mathrm{~mm}$; (f)

$$
D=0.8 \mathrm{~mm}, \alpha=0^{0}, \quad l=5 \mathrm{~cm}
$$

The mass loss of the cavitation was also measured by an electronic balance with a range of $10 \mathrm{~g}$ and a precision of $1 \mathrm{mg}$. Table 4 lists the measured accumulated mass losses in the present test. The accumulated cavitation mass loss in No. 9 test was greatest, suggesting best cavitation effect. The corresponding level combination of three factors is $D=1 \mathrm{~mm}, \alpha=30^{\circ}$ and $l=3 \mathrm{~mm}$. By analyzing the calculated range, the outlet angle most significantly affected the test results, followed by the hole diameter while the necking length imposed slightest effect. 
Table 4. Orthogonal test results

\begin{tabular}{|c|c|c|c|c|c|}
\hline \multirow{2}{*}{ Test number } & \multicolumn{4}{|c|}{ Factors } & \multirow{2}{*}{ Accumulated quality loss M/ (mg) } \\
\hline & D & & $\alpha$ & $l$ & \\
\hline 1 & 1 & 1 & 1 & 1 & 9 \\
\hline 2 & 1 & 2 & 2 & 2 & 89 \\
\hline 3 & 1 & 3 & 3 & 3 & 48 \\
\hline 4 & 2 & 1 & 2 & 3 & 44 \\
\hline 5 & 2 & 2 & 3 & 1 & 56 \\
\hline 6 & 2 & 3 & 1 & 2 & 12 \\
\hline 7 & 3 & 1 & 3 & 2 & 71 \\
\hline 8 & 3 & 2 & 1 & 3 & 20 \\
\hline 9 & 3 & 3 & 2 & 1 & 110 \\
\hline $\mathrm{K}_{1}$ & 146 & 124 & 85 & 175 & \\
\hline $\mathrm{K}_{2}$ & 112 & 165 & 204 & 172 & \\
\hline $\mathrm{K}_{3}$ & 201 & 170 & 175 & 112 & \\
\hline $\mathrm{k}_{1}$ & 48.67 & 41.33 & 28.33 & 58.33 & \\
\hline $\mathrm{k}_{2}$ & 37.33 & 55 & 68 & 57.33 & \\
\hline $\mathrm{k}_{3}$ & 67 & 56.67 & 58.33 & 37.33 & \\
\hline Extremum R & 29.67 & 15.34 & 39.67 & 21 & \\
\hline
\end{tabular}

Notes: $K_{\mathrm{i}} \quad(i=1 \sim 3)$ is the sum of the accumulated mass loss at level $i$ of these factors, $k_{\mathrm{i}}=K_{\mathrm{i}} / 3$.

\section{Conclusions}

By combining numerical simulation and test, this study analyze the effects of the structure of the nozzle in the net cleaning equipment under submerged environment on the inception, growth and collapse characteristics of the cavitation, and clarified the correlation between the cavitation erosion form and the nozzle's structural parameters. Main conclusions are described below.

(1) Under submerged environment, the nozzle pressure dropped rapidly to the confining pressure when flowing through the neck so as to form jet v. On account of intensive shear action between high-velocity jet and the nozzle neck's wall, local low-pressure region was formed at the neck corner, in which the pressure was far below than those in other regions. Accordingly, the fluid was locally fractured and cavitation inception occurred. The maximum gas-phase volume fraction reached up to $37.5 \%$. The cavitation nuclei were strengthened in the backflow region around the nozzle outlet and the vortex ring that was formed under the shear action of the jet in the peripheral region. Since cavitation phenomenon mainly occurred in the peripheral region of the jet, the sample generally exhibited annular cavitation effect, and obvious erosion marks appeared at the dead center.

(2) Under same inlet pressure, as the nozzle pore diameter increased from $0.6 \mathrm{~mm}$ to $1 \mathrm{~mm}$, the maximum gas-phase volume fraction was enhanced by $8.53 \%$, and both inner and outer diameters of the cavitation erosion rings increased, accompanied with the increase of the cavitation area by $20.83 \%$. It can thus be concluded that the increase of the nozzle pore diameter was beneficial to the enhancement of erosion intensity and area under same boundary conditions. The outlet angle structure of the nozzle promoted the formation of jet backflow region and further strengthened cavitation effect. The increasing necking length in the nozzle steadily weakened cavitation effect. 
(3) As the outlet angle of the nozzle varied within a range of $0^{\circ} \sim 60^{\circ}$, the induced maximum accumulated mass loss was approximately $101 \mathrm{~m}$, which was much greater than the maximum accumulated mass losses under these other conditions ( $D=0.6 \sim 1 \mathrm{~mm}$ and $\mathrm{l}=3 \sim 7 \mathrm{~mm}$ ). Among these three factors (the diameter of the nozzle $\mathrm{D}$, the outlet angle $\alpha$ and the necking length 1 ), $\alpha$ imposed the most significant effect, followed by $\mathrm{D}$, and finally by $\mathrm{l}$.

Acknowledgments: The authors greatly acknowledge the financial support from National Key R\&D Program of China (Grant No. 2019YFD0900903) , Fund of Key Laboratory of Open-Sea Fishery Development, Ministry of Agriculture and Rural Affairs, P. R. China (Grant No. LOF 2019-05), Central Public-interest Scientific Institution Basal Research Fund, South China Sea Fisheries Research Institute, CAFS(Grant NO.2019TS17), modern agricultural industrial technology system (Grant No. CARS-47), Special fund for the construction of innovation team of modern agricultural technology system in Guangdong Province, as well as Central Public-interest Scientific Institution Basal Research Fund, CAFS(Grant NO. 2019XT0204).

\section{References}

[1] Javier G.; Manuel S.; Javier A. Analytical Model for Predicting the UCS from P- Wave Velocity, Density, and Porosity on Saturated Limestone. Appl. Sci.2019,9:5265.

[2] Madin J.; Chong V.C.; Hartstein N. D. Effects of water flow velocity and fish culture on net biofouling in fish cages. Aquac. Res. 2010,41:602-617.

[3] Islam G. M. N.; Tai S. Y.; Kusairi M. N. A stochastic frontier analysis of technical efficiency of fish cage culture in Peninsular Malaysia. SpringerPlus. 2016,5:1127.

[4] Gorlach-Lira K.; Pacheco C.; Carvalho L.C.T.; Melo Juniror H.N.; Crispim M.C. The influence of fish culture in floating net cages on microbial indicators of water quality. Braz. J. Biol. 2013,73:457-463.

[5] Congedo P.M.; Goncalves E.; Rodio M.G. About the uncertainty quantification of turbulence and cavitation models in cavitating flows simulations. Eur. J. Mech. B-Fluid. 2015,53:190-204.

[6] Sun Y.; Guan Z.; Hooman K. Cavitation in Diesel Fuel Injector Nozzles and its Influence on Atomization and Spray. Chem. Eng. Technol. 2019,42:6-29.

[7] Li D.; Liu S.; Wei Y.; Liang R.; Tang Y. Numerical investigation on transient internal cavitating flow and spray characteristics in a single-hole diesel injector nozzle: A 3D method for cavitation-induced primary break-up. Fuel. 2018,233:778-795.

[8] Jian L.; Xiwen L.; Zuti Z.; Xiaohui L.; Yuquan Z. Numerical investigation into effects on momentum thrust by nozzle's geometric parameters in water jet propulsion system of autonomous underwater vehicles. Ocean. Eng. 2016,123:327-345.

[9] Mingming Z.; Can K.; Haixia L.; Jinhao C. Waterjet Cavitation Simulation and Erosion Mechanism Analysis of ASTM134 Device. CHINA SURFACE ENGINEERING. 2018,31:133-142.

[10] Hutli E.; Nedeljkovic M.; Bonyár A. Dynamic behaviour of cavitation clouds: visualization and statistical analysis. J. Braz. Soc. Mech. Sci. 2019,41:281-296.

[11] Peng K.; Tian S.; Li G.; Huang Z.; Zhang Z. Cavitation in water jet under high ambient pressure conditions. Exp. Therm. Fluid Sci. 2017,89:9-18.

[12] Peng G.; Yang C.; Oguma Y.; Shimizu S. Numerical analysis of cavitation cloud shedding in a submerged water jet. J. Hydrodyn. 2016,28:986-993.

[13] Yang M.; Xiao S.; Kang C.; Wang Y. Effect of geometrical parameters on submerged cavitation jet discharged from profiled central-body nozzle. Chin. J. Mech. Eng-En. 2013,26:476-482.

[14] Tang H.; Wrobel L.C.; Barton I.E. Two-phase flow patterns in turbulent flow through a dose diffusion pipe. Nucl. Eng. Des. 2005,235:1001-1014. 
[15] Piscaglia F.; Giussani F.; Montorfano A.; Hélie J.; Aithal S.M. A MultiPhase Dynamic-VoF solver to model primary jet atomization and cavitation inside high-pressure fuel injectors in OpenFOAM. Acta. Astronaut. 2019,158:375-387.

[16] Dai X.; Wang Z.; Liu F.; Wang C.; Sun Q.; Xu C. Simulation of throttling effect on cavitation for nozzle internal flow. Fuel. 2019,243:277-287.

[17] Du C.; Andersson S.; Andersson M. The effect of cavitation on the estimation of fuel injection rates based on momentum flux measurements. Fuel. 2019,238:354-362.

[18] Yuan C.; Song J.; Zhu L.; Liu M. Numerical investigation on cavitating jet inside a poppet valve with special emphasis on cavitation-vortex interaction. Int. J. Heat Mass Tran. 2019,141:1009-1024.

[19] SuGuo S.; Guoyu W.; ChangLi H. A Rayleigh-Plesset based transport model for cryogenic fluid cavitating flow computations. Physics, Sci. China Phys. Mech. 2014,4:764-773.

[20] Hegedűs F.; Koch S.; Garen W.; Pandula Z.; Paál G.; Kullmann L.; et al. The effect of high viscosity on compressible and incompressible Rayleigh-Plesset-type bubble models. Int. J. Heat Fluid Fl. 2013,42:200-208.

[21] Blume M.; Skoda R. 3D flow simulation of a circular leading edge hydrofoil and assessment of cavitation erosion by the statistical evaluation of void collapses and cavitation structures. Wear. 2019,428:457-469.

[22] Peng K.; Tian S.; Li G.; Huang Z.; Yang R.; Guo Z. Bubble dynamics characteristics and influencing factors on the cavitation collapse intensity for self-resonating cavitating jets. Petrol. Explor. Dev+. 2018,45:343-350.

[23] Christopher E.B. Cavitation and Bubble Dynamics. New York: Oxfourd University; 2013.

[24] Guo G.; He Z.; Lai M.; Duan X.; Leng X.; Duan L.; et al. Optical experiment and Large Eddy Simulation on effects of in-nozzle stagnant air bubbles and diesel on near-nozzle spray structure variation in diesel injector. Fuel. 2019,255:115721.

[25] Ban Z.H.; Lau K.K.; Shariff A.M. Prediction of the bubble nucleation rate in a quasi-stable cavitating nozzle using 2D computational fluid dynamics and enhanced classical nucleation theory. Eng. Appl. Comp. Fluid. 2015,9:247-258.

[26] Peng G.; Shimizu S. Progress in numerical simulation of cavitating water jets. J Hydrodyn. 2013,25:502-509.

[27] Xiaofei L.; Yaxin D.; Yuning Z.; Ningning T.; Yuning Z. Retardant Effects of Collapsing Dynamics of a Laser-Induced Cavitation Bubble Near a Solid Wall. Symmetry. 2019,11:1051.

[28] Tao Z.; Bo D.; Xiaohong C.; Zhonghua Q.; Rui J.; Weizhong L. Spray characteristics of pressure-swirl nozzles at different nozzle diameters. Appl. Therm. Eng. 2017,121:984-991.

[29] Gopalan S.; Katz. J.; Knio. O. The flow structure in the near field of jets and its effect on cavitation inception. J Fluid Mech. 1999,398:1-43. 